\title{
Spiral DRAGNs
}

\section{Minnie Yuan Mao*}

Joint Institute for VLBI ERIC (JIVE)

E-mail: mao@jive.eu

Double Radio sources Associated with Galactic Nuclei (DRAGNs) are almost always hosted by elliptical galaxies. The existence of DRAGNs hosted by spiral galaxies defies our current understanding of galaxy formation. However, to-date, a handful of such sources have been observed to exist in the local Universe, including the recently discovered J1649+2635. We call these sources "spiral DRAGNs".

EXTRA-RADSUR2015 (*)

20-23 October 2015

Bologna, Italy

(*) This conference has been organized with the support of the Ministry of Foreign Affairs and International Cooperation, Directorate General for the Country Promotion (Bilateral Grant Agreement ZA14GR02 - Mapping the Universe on the Pathway to SKA)

\footnotetext{
* Speaker.
} 


\section{Background}

In the local Universe, large-scale double-lobed radio sources are almost always hosted by elliptical galaxies [13]. This is consistent with leading galaxy formation models that suggest both elliptical galaxies and extended radio sources are the result of mergers [5, 4]. The existence of DRAGNs (Double Radio sources Associated with Galactic Nuclei) [8] hosted by spiral galaxies defies our current understanding of galaxy formation. Nonetheless, there have now been a handful of spiral DRAGNs identified including J1649+2635 [12]. J1649+2635 is the best example to-date of a grand-design spiral galaxy associated with a double-lobed radio source (Spiral DRAGN).

\section{Radio Galaxy Zoo \& the importance of Citizen Science}

The first three spiral DRAGNs to be discovered were all found serendipitously $[9,10,7,6,1]$. J1649+2635 was the first spiral DRAGN to be discovered in a systematic search for such systems using morphological classifications from the citizen scientist project Galaxy Zoo [11]. Mao et al. (2015) found only one spiral DRAGN in the overlap region of SDSS and FIRST [3], which highlights the rarity of spiral DRAGNs. In an attempt to quantify the number density of spiral DRAGNs in the local Universe, we have embarked upon a citizen scientist project using Radio Galaxy Zoo (RGZ) [2] to search for more spiral DRAGNs. The relatively digestible concept of spiral DRAGNs, coupled with the prospect of "pretty pictures" has made the RGZ Spiral DRAGN project one of the most popular RGZ projects. As of October 2015, the citizen scientists have identified over 300 candidate spiral DRAGNs. Figure 1 shows two spiral DRAGN candidates that have been identified by RGZ citizen scientists.
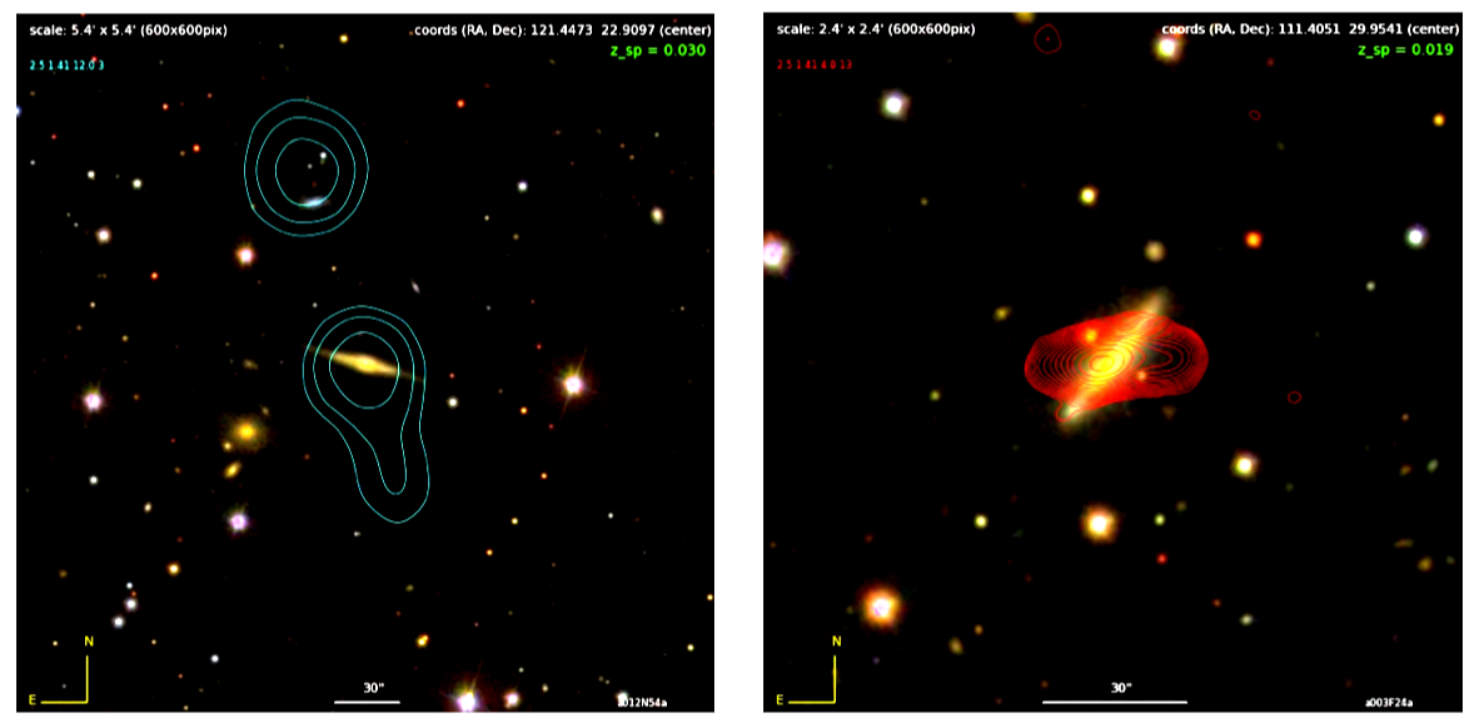

Figure 1: Candidate image overlays made by citizen scientist Jean Tate. The background images are from SDSS, red contours are from FIRST, and blue contours are from NVSS. 


\section{Probing the Nether Regions of Spiral DRAGNs with VLBI}

Do the black holes residing in spiral DRAGNs have special properties? Or do spiral DRAGNs reside in some particular environment? It is nature or nurture that is responsible for these rare, unexpected sources? We can study the radio properties of the AGN at VLBI-scales to answer these questions. Moreover, spiral galaxies are known to contain significantly more gas than elliptical galaxies. At VLBI resolutions we will be able to study any interaction the pc-scale jet may have with the host galaxy's ISM.

To date we have obtained VLBI data from the Long Baseline Array (LBA), Very Long Baseline Array (VLBA), and the European VLBI Network (EVN) for 0313-192 and J1649+2635. Both of these show VLBI-scale radio structure.

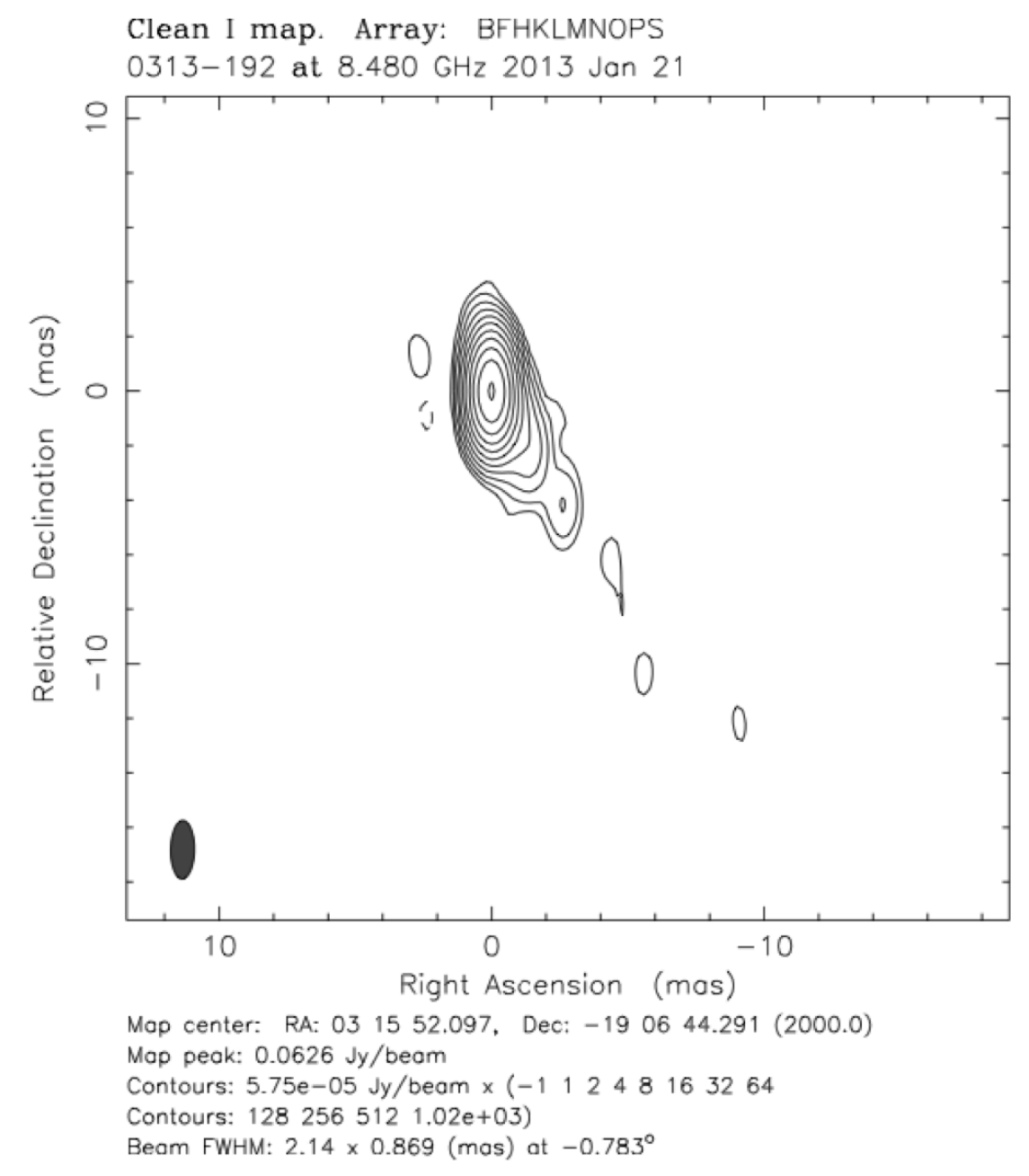

Figure 2: VLBA X-band image of 0313-192.

Figure 2 shows X-band data from the VLBA of 0313-192. The Southern jet has been detected. Interestingly, the angle of the jet is exactly aligned with the kpc-scale jet detected with the VLA suggesting that the jet is either powerful enough to have ploughed through the host galaxy's ISM, 
or that there was very little gas present for the jet to interact with.

\section{Current Status}

The standard galaxy formation model does not allow spiral galaxies to host large-scale DRAGNs. It is imperative that spiral DRAGNs are well-understood so as to establish their role in galaxy formation, either reconciling their existence with current models, or challenging the idea of the standard feedback model. Determining the prevalence of spiral DRAGNs is essential and it is only through deep, wide-field multi-wavelength surveys that these rare sources may be identified. Our team is working closely with next-generation surveys such as EMU [14] to optimise searches for unique classes of sources such as spiral DRAGNs.

\section{References}

[1] Bagchi J., et al., Megaparsec Relativistic Jets Launched from an Accreting Supermassive Black Hole in an Extreme Spiral Galaxy, ApJ, 788, 2014

[2] Banfield J. K., et al., Radio Galaxy Zoo: host galaxies and radio morphologies derived from visual inspection, MNRAS, 453:2326, 2015

[3] Becker R. H., White R. L., Helfand D. J., The FIRST Survey: Faint Images of the Radio Sky at Twenty Centimeters, ApJ, 450, 559, 1995

[4] Chiaberge M., Gilli R., Lotz J. M., Norman C., Radio Loud AGNs Are Mergers, ApJ, 806, 147, 2015

[5] Hopkins P. F., Hernquist L., Cox T. J., Kereš D., A Cosmological Framework for the Co-Evolution of Quasars, Supermassive Black Holes, and Elliptical Galaxies. I. Galaxy Mergers and Quasar Activity, ApJS, 175, 356, 2008

[6] Hota A., et al., Discovery of a spiral-host episodic radio galaxy, MNRAS, 417, L36, 2011

[7] Keel W.CJ., White III R. E., Owen F. N., Ledlow M. J., The Spiral Host Galaxy of the Double Radio Source 0313-192, AJ, 132, 2233, 2006

[8] Leahy J. P., Jets in Extragalactic Radio Sources, LNP, 421, 1, 1993

[9] Ledlow M. J., Owen F. N., Keel W. C., An Unusual Radio Galaxy in Abell 428: A Large, Powerful FR I Source in a Disk-dominated Host, ApJ, 495, 227, 1998

[10] Ledlow M. J., Owen F. N., Yun M. S., Hill J. M., A Large-Scale Jet and FR I Radio Source in a Spiral Galaxy: The Host Properties and External Environment, ApJ, 552, 120, 2001

[11] Lintott C. J., et al., Galaxy Zoo: morphologies derived from visual inspection of galaxies from the Sloan Digital Sky Survey, MNRAS, 389, 20081179

[12] Mao M. Y., et al., J1649+2635: a grand-design spiral with a large double-lobed radio source, MNRAS, 446, 4176, 2015

[13] Matthews T. A., Morgan W. W., Schmidt M., A Discussion of Galaxies Indentified with Radio Sources, ApJ, 140, 35, 1964

[14] Norris R. P., et al., EMU: Evolutionary Map of the Universe, PASA, 2011 